\title{
Sex and the cervix: hormones, infections and cancers
}

\author{
Lindsay Edouard
}

\section{Overview}

The link between contraception and sexually transmitted infections (STIs), including HIV, constitutes a recurring theme and more emphasis is needed on the incorporation of the control of those infections in the provision of hormonal contraception through the promotion of dual method use. Confirmation of the vital role of infection with the human papillomavirus (HPV) in the aetiology of cervical cancer led to innovative approaches: secondary prevention through detection of infection with HPV to complement established cytology screening and, lately, primary prevention of cervical cancer through vaccination against the most important subtypes of HPV.

\section{Scope of sexually transmitted infections}

Although cervical cancer is a largely preventable condition, it is the commonest cause of cancer mortality for women in developing countries. Far from being merely the gateway to the south in the female reproductive system, the cervix has an important role in the transmission of infections.

Observation of an increased mortality from cervical cancer among married women and the rarity of cervical carcinoma among nuns led, much later on, to the demonstration of a strong association of cervical carcinoma with several socioeconomic factors of deprivation. With epidemiological analyses not linking childbearing to the strong 'poverty' trend in cervical cancer, coital experience and penile hygiene were implicated. It was not until the mid-1970s that cervical cancer was suspected to be sexually transmitted ${ }^{1}$ and it was only in the 1980s that HPV was specifically incriminated.

By 1980, the concept of dual method use was already present for promoting both effective contraception and the prevention of STIs, as exemplified by the recommendation for the concurrent use of microbicides with the intrauterine device (IUD). Used for their microbial activity, Penotrane ${ }^{\circledR}$ pessaries contained hydrargaphen, an organic mercurial compound. Undue concern regarding a possible drug interaction led to the contraindication, on purely hypothetical grounds, of the use of Penotrane in conjunction with copper IUDs. It was felt that a reaction between copper and hydrargaphen could decrease both the contraceptive effectiveness of the copper IUD and antimicrobial properties of Penotrane. ${ }^{2}$

\section{Cervical squamocolumnar junction}

As "the custodian of the upper female genital tract", the cervix "commanded a greater level of respect and understanding" around 1980 besides being "the most studied organ in the female genital tract but also the most abused" and its secretions were thought to prevent the ascent of vaginal infections. ${ }^{3}$ The squamocolumnar junction had been shown to be the usual site of origin for

J Fam Plann Reprod Health Care 2005; 31(4): 338-339

\section{Larchmont, USA}

Lindsay Edouard, FRCOG, MFFP, Member of the Editorial Advisory Board

Correspondence to: Professor Lindsay Edouard. E-mail:soranae@gmail.com both physiological squamous metaplasia from oestrogens during pregnancy and adolescence and squamous cell carcinoma of the cervix. At the time, "a venereally transmitted carcinogenic substance" was incriminated, with the mutagen being "released by the degradation of sperm on the cervical epithelium or may be contained in one or more of the other sexually transmitted substances such as the Herpes simplex type II or human papilloma viruses." 3

\section{Revival of barrier methods}

Diaphragms and spermicides were "enjoying a slight revival, if one can judge from clinical records" around 1980.4 As barrier methods are less effective than hormonal ones for contraception, spermicides were highly recommended in conjunction with the diaphragm for dual method use. The October 1980 issue of the British Journal of Family Planning carried an advertisement for Genexol ${ }^{\circledR}$, the spermicidal suppository, with a quotation from Shakespeare to "make assurance double sure" (Macbeth, Act IV, Scene i).

Service providers should be aware of the importance of behavioural factors in dual method use. ${ }^{5}$ Consistent and correct use of the male latex condom is effective for protection against STIs such as HIV, gonorrhoea, chlamydia, herpes simplex virus type 2 (HSV-2) and syphilis. ${ }^{6}$ It is worrying that condom users often discontinue their use with uptake of hormonal contraception. It is plausible that consistent use of condoms is substantially higher among those using them, as opposed to hormonal methods, primarily for contraception. As nonoxynol-9, a moderately effective spermicide, was thought to be also valuable for the prevention of STIs, it was used for the lubrication of condoms during the manufacturing process. However, nonoxynol-9 leads to disruption of the vaginal epithelium and it was subsequently feared that its frequent use might increase the risk of acquisition of HIV. With evidence that nonoxynol-9 failed to reduce the risk of STIs, including $\mathrm{HIV}$, in high-risk populations, condoms lubricated with nonoxynol-9 are no longer promoted. ${ }^{7}$

\section{Hormonal contraception}

Besides causing cervical ectopy, sex hormones could increase the risk of cervical infections through the higher vaginal $\mathrm{pH}$ that occurs with a decrease in lactobacilli that produce hydrogen peroxide. Also, sex hormones might either increase the infectivity of certain infectious agents or suppress the immune system with local humoral dysfunction of the cervix. Progestogens cause both irregular bleeding and thinning of the vaginal epithelium. Finally, there has been concern that hormonal contraception might increase HIV transmission through its increased genital shedding in vaginal and cervical secretions. ${ }^{8}$

With the ethical imperative to provide individuals with their choice of a contraceptive method, it is impossible to use a randomised controlled trial as the experimental design to determine causality in the role of hormonal contraception in the acquisition of STIs: an observational prospective cohort study is the most likely approach. Major limitations of studies usually consist of the relatively small number of subjects, inappropriate characteristics of the comparison group and the 
differential risk of infection for the various subgroups. It is therefore difficult, albeit impossible, for statistical analyses to control for confounding factors. Furthermore, the findings might not be applicable beyond the study population: the risks for a specific outcome are usually different for sex workers, patients attending genitourinary clinics and individuals using family planning services, specially as pertaining to the number of partners and behavioural bias in the selection and utilisation of a contraceptive method. The crucial confounding factor might well be the very one that was not thought of. It is reassuring that hormonal contraception was not associated with an increased overall risk for the acquisition of HIV in a recent international multicentre prospective cohort study ${ }^{9}$ of about 6000 family planning clinic attendees, although there was an increased risk associated with HSV-2 seronegativity.

\section{Cervical carcinoma}

Cervical carcinoma has an infectious aetiology with the demonstration of the primordial role of HPV, which is present in more than $99 \%$ of cases. As HPV is present on the male and female external genitalia, barrier methods have a limited role in preventing transmission. Being largely asymptomatic, HPV infects, albeit transiently, about two-thirds of sexually active women. There is no treatment for microscopic infection by HPV, which may continue to be infectious for a number of years. Types 16 and 18 account for more than two-thirds of cases of cervical cancer, addition of types 45,31 and 33 increasing the proportion to $83 \%$. HPV 6 and 11 types are nononcogenic but lead to genital warts.

Whilst cytological testing continues to be the method of choice for cervical cancer screening, alternative modalities incorporating both HPV and cytology should be investigated. In poor-resource settings, visual tests can be performed by inspecting the cervix after staining with either acetic acid or Lugol's iodine, precancerous lesions turning white or yellow-on-brown, respectively. The iodine method, also referred to as Schiller's test in recognition of its use prior to the introduction of the Papanicolaou smear, seems to be more accurate than acetic acid which is more readily available specially in the form of vinegar.

Prophylactic, as compared to therapeutic, HPV vaccines provide protection against new persistent infections and the subsequent development of cervical intraepithelial neoplasia. Those vaccines will be introduced into service delivery in the very near future and are likely to have their maximal impact when administered before sexual debut. Therefore, cervical cancer screening will continue to be needed for at least a few more decades until the availability and uptake of multivalent vaccines that are active against the most important oncogenic HPV types as well as demonstration of the long-lasting effectiveness of those vaccines.

\section{Implications for services}

Hormonal contraception represented a major medical breakthrough in the second half of the last century. Unfortunately, decades after its introduction, it continues to be enrobed by numerous misconceptions that could be increased by misinterpretations of results from research studies. Despite their limitations, risk estimates for the acquisition of STIs can be useful for counselling purposes in guiding the utilisation of a hormonal contraceptive method.

Condoms provide dual protection from both pregnancy and STIs. But for those preferring the excellent contraception offered by hormonal methods, dual method use should be promoted for the complementary value of condoms in preventing infections.

A reproductive health approach should be used for the introduction of HPV vaccine to ensure excellent coverage of individuals before their initiation to sexual intercourse. Male vaccination has the potential to decrease female exposure to HPV, thereby accentuating the primary prevention of cervical cancer.

Statements on funding and competing interests

Funding. None identified.

Competing interests. None identified.

References

1 Beral V. Cancer of the cervix: a sexually transmitted infection? Lancet 1974; 1: 1037-1040.

2 Morton O. Penotrane pessaries and IUCDs. Br J Fam Plann 1980; 6: 27 .

3 Mylotte MJ. Sex and the cervix. Br J Fam Plann 1980; 5: 86-89.

4 Jackson M. The changing face of family planning. Br J Fam Plann 1980; 6: 71-74

5 Bostock Y, Hunter JM, Roberts JE. Patterns of sexually transmitted diseases in young people and implications for health education. $\mathrm{Br}$ J Fam Plann 1980; 6: 82-85.

6 Holmes KK, Levine R, Weaver M. Effectiveness of condoms in preventing sexually transmitted infections. Bull World Health Organ 2004; 82: 454-461.

7 World Health Organization/CONRAD. WHO/CONRAD Technical Consultation on Nonoxynol-9: Summary Report. Document WHO/RHR/03.08. Geneva, Switzerland: WHO, 2003: 14.

8 Okonofua FE. Rethinking contraception in Africa in the era of HIV/AIDS. Afr J Reprod Health 2004; 8: 7-9.

9 Morrison CS, Richardson BA, Celentano DD, Chipato T, Mmiro F, Padian NS, et al. The hormonal contraception and risk of HIV-1 acquisition (HC-HIV) study: background results and discussion (Abstract TW-002). 16th Biennial Meeting of the International Society for Sexually Transmitted Diseases Research, 10-13 July 2005, Amsterdam, The Netherlands.

\section{LETTERS TO THE EDITOR}

Letters to the Editor are welcome and generally should not exceed 600 words or cite more than five references. For comments on material published in the most recent issue of the Journal, correspondence should be received within 4 weeks of dispatch of that Journal to be in time for inclusion in the next issue. When submitting letters correspondents should include their job title, a maximum of two qualifications and their address(es). A statement on competing interests should also be submitted for all letters. Letters may be submitted to the Editor or the Journal Editorial Office (details on page 261). 